\title{
Surrogates of Recognition. On the Reconstruction of a Possible Critical Hegelian Contribution to Current Discussions on "Identity"
}

\section{Anerkennungssurrogate. \\ Zur Rekonstruktion eines möglichen kritischen Hegelschen Beitrags zu heutigen Diskussionen über ,Identität}

\author{
Rainer Adolphi \\ (Technische Universität Berlin; rainer.adolphi@tu-berlin.de)
}

\begin{abstract}
The article discusses a central topic of contemporary understandings of society that seems to have no place in Hegel's theory: the topic of "identity", which seems to fall between the process of a "struggle for recognition" on the one hand, and, on the other, a consolidated recognition of subjects and their rights within the established social order. The article would like to propose a further reconstruction here. It discusses which considerations should be included so that the discourse on "identity" does not end in any substantialist or ethno-national, egocentric understandings, but, instead, could become possibly a part of Hegel's theory. In today's dynamics and unsettling changes, there are undeniable needs for "identity" (which are also easily addressed, even fuelled, by corresponding offers). These are, as one could learn from Hegel, surrogates of a still not or no longer successful sufficient recognition. In this, "identity" is to be understood as critical work on oneself as a product of becoming, on inheritances, achievements, challenges, divisions, discrepancies, guilt and failures.
\end{abstract}

Keywords: Hegel; identity; modernity; recognition; politics of history.

Ethik macht man, wenn man Ethik braucht. Fast stets verlaufen die Entwicklungen so, dass erfahrene Wirklichkeiten auf Prozesse und Aspekte deuten, die bisher noch nicht (oder nicht eigens) reflektiert waren. Durch erfahrene, bewusstgewordene Wirklichkeiten kommen neue Wichtigkeiten in den Blick. In dieser Hinsicht geht es der philosophischen Ethik nicht anders als der psychologischen Modellbildung oder der soziologischen Theorie. Und auch inhaltlich sowie konzeptionell sind im Konkreten die Schübe der Entwicklung oft vermittelt über psychologische, sozialwissenschaftliche, anthropologische Betrachtungen und Erkenntnisse. Allgemein ist die Entwicklung sowohl Ausdifferenzierung und Spezifizierung als auch die Erweiterung von Fragestellungen und mit einbezogenen Sachverhalten. Umgewichtungen in den ethischen Wertungs- 
Perspektiven und im Themenfokus der Debatten ergeben sich meist sekundär von dort her.

So gibt es denn heutige Kernthemen der Ethik, über die etwa Kant, Fichte oder Hegel nicht reflektiert haben. Dazu gehören das Thema der, Werte', das Thema des ,Sinns', Thema der personalen (existenziellen) Authentizität, Thema der ,Verantwortung', Thema von Ethik und Technisierung der Welt, Thema der ,Lebenskunst', überhaupt Lebensgeschichte, Thema der ,Identität', und nicht zuletzt die neuen Fragen des ,Bösen' (Aufbrechen eines „Bösen' im Zustand hoher Kultur), Phänomene der Wiederkehr stoizistischer Haltungen und die Phänomene einer neuen Spiritualität. Die frühere ethische Reflexion, bes. in der klassischen Epoche von Kant, Fichte und Hegel, dachte unter Zugrundelegung einer Parallelität von individueller und gesellschaftlicher Entwicklung - Perspektive eines (mit Moderne konnotierten) Bildungsprozesses in Parallele von Individuen (in Denken, Empfinden und Handeln) und Gesellschaftsganzem. Alle seitherigen erweiternden Themen liegen gewissermaßen an dieser Bruchlinie zwischen Individuellem und Gesellschaftlichem.

Das Folgende möchte in den heutigen gesellschaftlichen Verständigungen vielleicht zum wichtigsten gewordene Thema ins Zentrum stellen: das Thema der ,Identität'. Erörtert werden soll es im Horizont von Hegel. Das Thema scheint in Hegels Theorie keinen Ort zu haben. Es scheint hindurchzufallen zwischen einerseits den Prozessen eines „Kampfes um Anerkennung“, die Hegel als gleichsam unmittelbares Gegenüber von Personen oder Gruppen in ihrem beiderseitigen, polar-bezogenen „Selbst“ erkannt hatte (Hegel 1807, 104-128), ${ }^{1}$ und andererseits dem gefestigten "Anerkanntsein“ (vgl. Hegel 1830, §§ 432f./484; auch Hegel 1830, §§ 490/527, Hegel 1821, §§ 192/217) von Subjekten und Rechten innerhalb der etablierten, modernen „sittlichen“ Ordnung. Die soziale und politische Ordnung der „Sittlichkeit“ im Sinne der Hegelschen Theorie scheint neutral zu sein bezüglich Fragen der ,Identität', auch Erfahrungen der Verunsicherung der ,Identität' und überhaupt Verunsicherungen des Geschichtsbewusstseins. - Das Folgende möchte hier eine weiterführende Rekonstruktion vorschlagen. Es soll erörtert werden, welche Reflexionen aufgenommen werden müssen, damit die Rede von ,Identität' nicht unwillkürlich in (konstruierten) substanzialistischen Verständnissen und nationaler (oder ethnischer, religiöser) Eigenzentrierung endet, sondern zu einem Teil gerade einer hegelianischen Theorie werden könnte. ,Identität' soll dabei nicht statisch und rein affirmativ verstanden werden, sondern als Arbeit - oft erforderlich auch kritische Arbeit - am eigenen Gewordensein, an Erbschaften, Errungenschaften, Bezügen-zu-Anderem, aber ebenso an Problemen, Schuld und Versäumnissen.

Hegel in seiner Zeit, zwischen historistischen, nationalistischen, bildungselitären, konfessionalistischen und antisemitischen Strömungen und den Behauptungskämpfen der alten Stände-Ordnung, hatte gute Gründe, das Thema - und ,wer' Träger der wahren

1 Kap. IV.A.: Selbständigkeit und Unselbständigkeit des Selbstbewußtseins; Herrschaft und Knechtschaft. 
„sittlichen“ Gemeinschaft ist - fernzuhalten von den Ausführungen seiner Theorie. In den Konstellationen seiner Zeit waren Diskurse über ,Identität' Legitimierung der ,guten alten Herrschaftsformen und blinder Nationalismus, gegenüber den Ideen der Französischen Revolution und auch Forderungen wie der der Judenemanzipation. Unter heutigen Gegebenheiten starker Dynamik und verunsichernden Wandels aber gibt es unleugbar ,Identitäts'-Bedürfnisse - durch die faktischen Gegebenheiten bedingte Bedürfnisse, die auch von entsprechenden Angeboten leicht anzusprechen, ja aufzuheizen sind. ${ }^{2}$ Dazu eine Betrachtung in drei Teilen.

\section{Modernisierungserfahrungen und Identitätsbedürfnisse (Zur geschichtlichen Konstellation des Themas der ,Identität')}

(1) Amartya Sen hat auf das Problem aufmerksam gemacht, dass Gemeinschaften, und gerade zunehmend wieder, sich in singularisierten und einander ausschließenden ,Identitäten' verstehen. Es ist ein Problem, das in der neuen globalen Konstellation nach dem Ende der großen 2-Teilung der Welt, deren Fundamentalpolarisierung das 20. Jahrhundert vom Ersten Weltkrieg bis zu den Umbrüchen von 1989/90 beherrscht hatte, noch einmal besonders sich verstärkt. In der Tat breitet sich in vielem eine Kriegsrhetorik aus. Nationen und Kulturen empfinden sich im Kampf um ihre jeweilige Wesensidentität, die durch ,Fremdes' bedroht werde. Es ist ein Denken nach der Logik der Verdrängung: Wo das Eine ist, könne das Andere nicht sein, nicht existieren.

Jede Gemeinschaft, jede Zugehörigkeit - das ,Wir' bzw. ,das Fremde - wird als eine einzige, ganzheitliche Identität (Identitätsgruppe) gedacht, der die Einzelnen in ihrem ganzen Wesen zugeordnet sind bzw. sich zuordnen müssen. Dass Verschiedenes in einem gemeinsamen Raum, Tür an Tür, friedlich und (geschichtlich-)versöhnlich als Nachbarn zusammen leben könnte, ist in diesem Denken nicht vorgesehen. ${ }^{3}$ Es ist von vornherein ausgeschlossen. Beide jeweilige Seiten seien, für die konkreten Menschen unabänderlich schicksalhaft, zur Ausschließlichkeit ${ }^{4}$ bestimmt (Sen 2006). - Ängste vor einem vorgestellten Anderen oder Verändernden werden da dann allzu leicht zu xenophoben Grundmentalitäten und richten sich gegen die vermeintlichen ,Fremden: Solches Denken betont einseitig das Trennende statt des Verbindenden (oder erforderlich

2 Meine Erörterungen in diesem Artikel mögen gesehen werden als eine Reflexion in dem, was Jakub Kloc-Konkołowicz und Marek Siemek als Feld einer Forschungsaufgabe hinterlassen haben. Im Geist ihrer Erbschaft gilt es heute, die Tradition idealistischer Philosophie zu verteidigen gegen zumal deren Umdeutungen aus, romantischen' Denkorientierungen des 19. Jhs. wie auch aus szientifischen Depotenzierungen aller Fragestellungen. Dies wird - wie hier beim Thema der ,Identität ${ }^{\prime}$ - nicht selten auch erkennen müssen, wie Hegelsche Gedanken, wenn sie später in den verschiedenen Hegel-Rezeptionen (und -Renaissancen) tragend wurden, oft in Verschmelzung mit anderen Konzeptionen gebracht sind, gemeinhin nicht zum Vorteil an argumentativer Stärke und Klarheit.

3 Ebensowenig, dass in einem Individuum oder einer Teilgruppe mehrere lebensbedeutsame Zugehörigkeiten zusammenkommen können, sich verschränken können.

4 Oder allenfalls zeitweise strategische Koalitionen - gegen Dritte. 
Transformativen). Indem in den Vorstellungen der Menschen das verstärkt wird, was sie in Wesensidentität von anderen abhebe und trenne, ist dem Verbindenden und der Suche eines Gemeinsamen und des Ausgleichs immer weniger eine reale Chance gegeben, eine Chance in der Weise des Zusammenlebens. Und dort erwachsen daraus immer größere Gefahren, Geschichte zu missbrauchen, um mit entsprechenden Geschichts-Konstruktionen ein identitäres ,Wir'-Sein (oder komplementär eine Erbgegnerschaft) zu untermauern.

Die Phänomene, die hier zu diagnostizieren sind, werden immer offenkundiger, und zunehmend in allen Gesellschaften. Das enthebt jedoch nicht der Frage, woher die Bedürfnisse kommen, sich über volks-, nationen- und kulturhafte ,Identitäten zu bestimmen, ja sich daran zu klammern. - Dass dies so hoch besetzt ist, ist sicher nicht $\mathrm{zu}$ verstehen ohne die motivierenden Hintergründe: nämlich woher die Verunsicherungen, nicht (oder nicht mehr) heimisch zu sein im Eigenen, kommen; woher das Unsicherheitsgefühl, das die Sicherung einer ,Identität' - und die geistige Versicherung darüber - so herausstreichen, d.h. so sehr zum sozialen und politischen Argument werden lässt. Alte Gesellschaften und frühere Jahrhunderte waren so gut wie immer identitätsverschlossen und insofern allophob - weil sie mit gar nichts anderem in Berührung kamen als mit sich selbst. Abgrenzungen waren ihnen nur wichtig gegen das vorsoziale Sein, vorsoziale Leben. Doch unter modernen Bedingungen und translokalen Horizonten haben die Prozesse eine besondere Bedeutung. Sich heute über volks-, nationen- und kulturhafte ,Identitäten' zu bestimmen, hat entscheidend auch eine mentale Seite der spezifischen Identitätserwartungen.

(2) Diskurse der ,Identität' sind eine Antwort - doch worauf? In einer ersten Annäherung ließe sich sagen: Von ,Identität' kann man sinnvollerweise nur und erst dann sprechen, wo es Alternativen, sich selbst und das eigene Zusammenleben zu sehen wie zu erfahren, gäbe, anderes, als sich über vorgestellte fixierte ,Identitäten' zu bestimmen. Oder wo man das Mächtigwerden dieser Alternativen als drohend empfindet.

Natürlich gibt es Kultur- und auch Sprach-Entwicklungen, die wie feindlich wirken; so z.B. genau in dem historisch durch so plurale Regionen gekennzeichneten Europa, wo das vormals durch Flüsse, Wälder, Gebirge und Religionen Separierte immer stärker in Kontakt kommt. Aus dem Zusammenwachsen der (Lebens-) Räume, aus der Mobilität, aus der Verflechtung in übergreifende ökonomische Prozesse - sowie nicht zuletzt aus dem Wissen, den wechselseitigen Informationen - ist oft noch keine entsprechende mentale Gemeinschaftlichkeit, Brüderlichkeit und Solidarität geworden. Das Mentale und die Empfindungen der Menschen hinken bei großen Veränderungen meist erheblich hinterher. Und die innereuropäischen Arbeits-Ströme sowie die globalen Migrationen haben vielerorts die Vertrautheit, nur mit angestammt ,Gleichen' und Zugehörigen zusammenzuleben, in einer zu schnellen Weise belastet.

Unvertrautheiten haben jedoch oft - mit Nähe zur Gegenwart dies auch immer stärker - nicht eigentlich mit einer Bedrohung der ,Identität' durch andere Menschen, eine andere Kultur, andere Mächte zu tun. Sondern sie erwachsen aus dem sozusagen 
gemeinsamen Schicksal der Modernisierung, der gemeinsamen Verstrickung in die Faktizitäten der Modernisierung. Durch Modernisierungsentwicklungen, ihren übergeordneten Druck aufalles, kommtes auf allen beteiligten Seiten zu einem Bruch in den Selbstverständlichkeiten von Lebensformen und Arbeit, zu einer Erosion der jeweiligen kulturellen Normtraditionen und Bindungen, die nicht schnell genug nachrücken können, zu Spannungen im Verhältnis der Generationen. Dies sind allem voran jeweilige innere Verwerfungen der Gesellschaften. Es gibt immer Gruppen - meist die Jüngeren, die Gebildeteren, die Stadtbevölkerung -, die sich den Veränderungen schnell anpassen, ja bezüglich ihres Status nutzen können. Und es gibt immer Regionen, Bevölkerungsgruppen, Tätigkeitsfelder und generationentypische Biographien wie Erfahrungen, für die die Macht (Übermacht) der Veränderungen und ihre kalte Unerbittlichkeit nicht nur reale Einbußen und Deklassierungen bedeuten, sondern stets auch narzisstische Kränkungen so, als werde einem die Identität genommen. Das Vorgestellte, dass es so kommen könnte, ist hier subjektiv und sozial oft noch wirkungsvoller als das tatsächlich Eintretende.

Diese Ängste vor dem gesichtslosen Anderen, Gesichtsloses im Eigenen, sind es, was - sozusagen negativ - heute den Hauptteil des Verlangens nach einer klaren, einfachen ,Identität' und deren Sicherheit speist. Je rigider eine Behauptung der Identität eingefordert wird, desto mehr sind das meist Kompensationen. Es kommt zur Bereitschaft, sich ,Identitäts'-Angebote zu eigen zu machen und deren Wortführern Gefolgschaft zu leisten. Es erwachsen Projektionen auch innerhalb des bisherigen Zusammenlebens, Projektionen eines auch inneren Feindes, der mit den Mächten der Bedrohung paktiere - ein Ruf nach Identitätspolitik der,wahren' Gemeinschaft, Ruf nach energischen Maßnahmen und ein gärender innerer Kulturkampf, Kulturkampf im Eigenen. Darin werden denn auch Machtkämpfe der ,Identitäts'-Propaganda ausgetragen, Kämpfe der Deutungsführerschaft. Und allgemein leben die Menschen unversehens in einem Klima des Drucks der Überidentifikation.

Die Rede von ,Identität', Diskurse über ,Identität' sind dabei gänzlich ohne Selbstreflexion. Sie verschärfen in allem nur die Ängste, statt konkrete Ursachen zu benennen.SieanonymisierenallesErfahreneerstrecht.MitModernisierungsentwicklungen gibt es offenkundig große - und immer neue - Integrationsaufgaben, doch hier sind sie abgebogen; die Kausalitäten erscheinen als verschoben bzw. umgedreht. Imaginierte Furcht und Bedrohung potenziert dann nur real erfahrene Kränkungen und die Anforderungen der Veränderung. Gerade in der dynamisierten Moderne werden dadurch Sicherheitserwartungen geschürt, die die Menschen in vormodernen Zeiten so gar nicht hatten; und dies lässt sich oft leicht politisch instrumentalisieren. -

Soweit zunächst die zeitdiagnostische Phänomenalität. Sie führt zur Notwendigkeit eines allgemeineren Blicks und damit auch zum Bezug auf Hegel: auf das, was, in entsprechender weiterführender Rekonstruktion, eventuell von Hegel zu lernen wäre. 


\section{Konstruktionen der ,Identität' (Figuren des Denkens in der Verständigung über ,Identität': eine Hegelsche Perspektive)}

Das Bewusstsein, welche Probleme das Konzept der ,Identität' birgt, ist im Grunde alt. Mit der Skepsis gegenüber metaphysischen Hypostasierungen hatte die Reflexion der Neuzeit schon früh erkannt, dass der Mensch, sofern er wesenhaft auch durch seine mentale ,Innenseite‘ und deren Geschehnisse gekennzeichnet ist, eine besondere Form von Identität besitzt, die nicht mit der generellen ontologischen Kategorie gedacht werden kann, nicht in selber Weise wie dinghafte (oder logische) Entitäten. J. Locke hatte in diesem Sinne die Identität eines Menschen an der Kontinuität seines Bewusstseins festgemacht (vgl. Locke 1694, Book II, Chapter XXVII). Jüngste Konzeptionen haben dies dann dahin ausformuliert, dass in Bezug auf Wirklichkeiten des Bewusstseins die Identität etwas relativ Eigenständiges ist gegenüber der physischen Seite als manifest Vorhandenem und dessen Kausalitäten, auch gegenüber der reinen Summe des einzelnen phänomenal Erlebten (bzw. seiner Gewissheiten von Seins-Gegenständlichkeiten wie -Sachverhalten): Die Identität von Mentalem ist narrativ verfasst ${ }^{5}$ und folgt durch Praktiken - primär bes. Einsozialisierung - vermittelten Formen, habituell geronnen in Weisen des gelebten Umgehens-mit.

Das 19. Jahrhundert, mit seinen aus der Romantik geborenen Ideen der ,Nation" und von ,Volk', hatte dies bereits auf kollektive Identitäten übertragen, dabei aber substanzialisiert. Das Wahre erschien als der Wesenscharakter eines Volkes bzw. einer Nation, welches feste, sich selbst empfindende Wesen aus den Ursprüngen entstamme, und gegenüber dem Entwicklung allenfalls negativ die Gefahr des Verlustes sein könne. Wenn seither und heute gesellschaftliche und mentale Probleme eines Zusammenlebens und der politischen Ordnung immer wieder als Probleme der ,Identität gezeichnet werden, wirkt darin meist irgendwo dieser Geist der Romantik. Die Vorstellungen bleiben im Bann eines Denkens des 19. Jahrhunderts. -

(1) Dies hat freilich nicht allein weltanschauliche oder ideologische Ursachen, Ursachen einer Geistesatmosphäre. ${ }^{6}$ Auch allgemeine strukturelle Arten des Denkens - und im Gefolge Muster der Theoriebildung - bei dem, Fragen der ,Identität' zu thematisieren, spielen dabei eine prägende Rolle, gerade auch unter heutigem Modernisierungsdruck.

Mit dem, was die klassische Tradition der Hegelschen Theorie als die „sittliche“ Weise, „sittliche“ Integration eines Zusammenlebens von Individuen in sozialen und politischen Gemeinschaften expliziert hat - „Sittlichkeit“ als Gemeinsames der normativen Alltäglichkeiten und Verbindendes des Verhaltens, erfahrungsbefestigte wechselseitige Erwartungen und Vertrauen, und zugleich als gelebte Zusprechung von entsprechender Subjektivität und Rechten ${ }^{7}$-, lassen sich erhebliche Felder dessen, was die Fokussierung

5 Vgl. dazu die grundlegenden Erörterungen von Ricœur (Ricoeur 1983-1985; 1990).

$6 \mathrm{Zu}$ einer besonderen Konstellation davon in Deutschland und Polen, lehrreich für die sowohl geistigen wie gesellschaftlichen Prozesse, die dabei schon im 19. Jh. Beginnen vgl. Adolphi 2019.

7 Zum konzeptionellen Kontext bei Hegel vgl. Adolphi 2018. 
auf ,Identität' in ihrer primären Evidenz so unreflektiert hat bleiben lassen, ja die ganze Sogkraft dieser Diskurse unter Gegebenheiten der Moderne, als Verkehrungen des Denkens in den gesellschaftlichen Verständigungen erkennen. Schon direkt hat Hegel bei der politischen Gemeinschaft des Staates einen der auratischsten Kernbegriffe der ,Identitäts'-Diskurse, die Forderung des „Patriotismus“ und seiner Vorordnung vor andere normative Wertkriterien, auf sein nüchternes Maß zurückverwiesen. ${ }^{8}$ Am Eingang des politischen Teils seiner grundlegenden Rechtsphilosophie heißt es - fast beiläufig -, dass die Momente, in denen die Bindung an die Nation von großen aufwallenden Emotionen ist, nicht der Standard sind. Vor allem darf sie, wie man das konkretisieren könnte, nicht etwa politisch missbraucht werden für Überheblichkeit und Auftrumpfen gegen andere und Aufforderung zu entsprechenden heroischen Aktionen, oder für die Diskreditierung des bürgerlichen Lebens. ${ }^{9}$ Die wahre (,sittliche“) Bindung beweist sich im Alltäglichen des selbstsicheren Habitus, loyal zu den Institutionen zu stehen und andere, ungeachtet von Differenzen, als Menschen und Mitbürger zu behandeln, nicht in der grimmigen oder gar fanatischen Haltung, niederschwellig bereit zur Abgrenzung, Konfrontation, Aggression. ${ }^{10}$ Die Einrichtung des politischen Gemeinwesens, sie zuvörderst, muss gut sein - d.h. damit die Bürger ihre Anerkennungen und die Geltung ihrer Rechte erfahren können -, die Einrichtung, nicht ein Pathos der Gesinnung; bei guter Einrichtung ergibt sich die Gesinnung, in ihrem relativ erforderlichen Maße, auf natürliche Weise von alleine. ${ }^{11}$

Ebenso koppelt Hegel - mit der Konzeption der „Sittlichkeit“ eines politischen Zustands - die Gemeinschaft, die Zugehörigkeit und den Status der Einzelnen, nicht

8 Analoges gälte für ihn erst recht für ethnische und auch religiöse Eigenverabsolutierung, Arroganz, Hochmut, Gefühlsaufwallung der Selbsterhöhung, Fanatismus.

9 Wie zuletzt der Vorstellung vom Krieg als dem ,Stahlbad' der Nation, als in gewissen Zeiträumen lebenserforderlich gegen die Mattigkeit und die pure Privatheit der Zwecke, in die das bürgerliche Leben immer wieder herunterzusinken neige. Bürgerliches Leben hat das Recht auf das Engagement in jeweiligen besonderen Interessenhorizonten (und zu entsprechender selbstverständlich-solidarischer gesellschaftlicher Anteilnahme wie Mitwirkung). Und übrigens auch ist das Militärische als ein eigener Berufsstand, die Souveränität gegebenenfalls nach außen zu sichern, einzurichten - Militärisches ist für Hegel nicht ins gesellschaftliche Leben hineinzutragen (vgl. Hegel 1821, §§ 322-328). (Hier gibt es freilich zugegebenermaßen Formulierungen, die aus Sicht heutiger Zeit missverständlich wirken können. Doch hat Hegel auch gelernt; extrem noch, nach dem Vorbild Platons, war die Absolutheit des Einheitsganzen und dessen Zwecke in seiner frühen Jenaer Zeit, bes. im Naturrechtsaufsatz.)

10 „Die politische Gesinnung, der Patriotismus überhaupt, als die in Wahrheit stehende Gewißheit (...) und das zur Gewohnheit gewordene Wollen ist nur Resultat der im Staate bestehenden Institutionen, als in welchem die Vernünftigkeit wirklich vorhanden ist, so wie sie durch das ihnen gemäße Handeln ihre Betätigung erhält. (...) Unter Patriotismus wird häufig nur die Aufgelegtheit zu außerordentlichen Aufopferungen und Handlungen verstanden. Wesentlich aber ist er die Gesinnung, welche in dem gewöhnlichen Zustande und Lebensverhältnisse das Gemeinwesen für die substantielle Grundlage und Zweck zu wissen gewohnt ist. Dieses bei dem gewöhnlichen Lebensgange sich in allen Verhältnissen bewährende Bewußtsein ist es dann, aus dem sich auch [bei begrenzten herausfordernden Anlässen] die Aufgelegtheit zu außergewöhnlicher Anstrengung begründet. Wie aber die Menschen häufig lieber großmütig als rechtlich sind, so überreden sie sich leicht, jenen außerordentlichen Patriotismus zu besitzen, um sich diese wahrhafte Gesinnung zu ersparen oder ihren Mangel zu entschuldigen“ (Hegel 1821, § 268).

11 „Auf die Frage eines Vaters nach der besten Weise, seinen Sohn sittlich zu erziehen, gab ein Pythagoreer (auch anderen [Hegels Handexemplar: „dem Sokrates“] wird sie in den Mund gelegt) die Antwort: wenn du ihn zum Bürger eines Staats von guten Gesetzen machst" (Hegel 1821, § 153). 
an einen hohen Begriff von (nationaler, gar ethnischer, religiöser) ,Identität' o. dgl. Am Beginn der Thematisierung der Rechtsordnung eines Gemeinwesens von Bürgern ist dazu in deutlichen Worten festgehalten: In einem wahrhaften „sittlichen“ Gemeinwesen, gleich wo in der Welt, gilt ein jedes „Ich als allgemeine Person (...), worin Alle identisch sind. Der Mensch gilt so, weil er Mensch ist, nicht weil er Jude, Katholik, Protestant, Deutscher, Italiener usf. ist" (Hegel 1821, § 209). ${ }^{12}$ Ein jeder ist Rechtsperson und im Sinne der Gesetze des bürgerlichen wie des staatlichen Rechts ohne Privilegien einzelner Stände (Adel, Patriziat, Kirche, ,Altbürger' in Bezug auf Vorrechte, Jurisdiktion oder Schuldverantwortung) und mit zugestandener moralischer Innerlichkeit.

Von diesem Horizont aus muss man denn wohl eingestehen, dass zunächst persönliche Verhältnisse des Hineingeborenwerdens und der ,familialen' Strukturen allenfalls in einer uneigentlichen Bedeutung von einer ,Identität' im Sinne der Diskurse getragen sind. Wie Hegel herausstreicht, beruhen diese Dimensionen - zumindest modern, wo der Holismus des Traditional-Herrschenden und der darin gesetzten Zuschreibungen wie geforderten Fügungen gebrochen ist - nicht auf einer blanken Verschmelzung; ${ }^{13}$ und das Generative vollzieht sich, sofern nicht durch umfassende altpatriarchale Autorität unterworfen, in der Ablösung der Heranwachsenden zur (willentlichen) Bildung eigener persönlicher Verhältnisse und Lebenszusammenschlüsse (vgl. Hegel 1821, $\S 177)$, und auch den bestehenden Herkunfts- und familialen Gemeinschaften sei eine Auflösung der Bindung zuzugestehen, wenn die gemeinsamen Lebensverhältnisse oder Gefühle nachhaltig zerrüttet sind (vgl. Hegel 1821, § 176). Persönliche Verhältnisse kraft Hineingeborenwerden, Herkunft und direkt personalen zwischenmenschlichen Gefühlen (in Hegels Begrifflichkeit: „natürliche Sittlichkeit“, „,natürlicher sittlicher Geist“) sind eine Gemeinschafts-Einheit, aber nicht eine darüber hinausgehende mystische ,Identität. ${ }^{14}$ Dann, die Gemeinschaftsformen der modernen ,arbeitsteiligen' Gesellschaft - Beziehungen und Vernetzungen - bilden erst recht keine qualitative ,Identität' ihres Ganzen („bürgerliche Gesellschaft“), sondern nur die möglichst allseits förderliche Aufrechterhaltung der funktionalen Prozesse. Die Gemeinschaften - Zugehörigkeiten, Werte und Wichtigkeiten - sind innergesellschaftliche Gemeinschaften im Plural, alle Einzelnen in vielfachen, einander überkreuzenden Kreisen stehend.$^{15}$ Die Anerkennungen

12 Immer wieder ist selbst in der Neuzeit „der Unterschied zur Ungebühr hervorgehoben worden, und man hat nach Juden und Christen, Engländern und Franzosen gefragt, mehr als nach dem Menschen" - dem Menschen, worin alle in gleicher Weise Bürger sind, anerkannt und mit Rechten (Hegel 1819/20, 169).

13 Wenn ich in eine schlechte, unverantwortlich schädliche Familie oder Clan-Formen hineingeboren werde, habe ich in der Gesellschaft - durch das Große der Gesellschaft - die Chance der Freiheit, anstatt die Setzungen und Ansprüche meiner Herkunftsgemeinschaft mein Lebtag mit mir tragen zu müssen.

14 Das schließt ,Identitäts'-Empfindungen im Sinne der Identität kleinräumiger Regionalitäten nicht aus, ja auch das (unaggressive) Engagement für deren Bewahrung. Aber auch seine Herkunftsregion und deren Eigentümlichkeiten muss man nicht nur lieben.

15 Wie später Georg Simmel dies bildlich ausdrücken sollte. - Hegel, in seiner Zeit, hat die Pluralität noch primär an den Lebens- und Tätigkeitsbereichen der Einzelnen festgemacht, wo sie als Mitglieder ihrer jeweiligen „Korporations“-Gemeinschaft ihre spezifische Anerkennung, Ehre 
gelten hier gerade einem Eigensein des Jeweiligen. Schließlich aber auch das politische Ganze, in der Verfasstheit seiner Ordnung, seinen gesinnungsmäßigen Grundlagen dem Geistig-Substanziellen der habituellen „politischen Gesinnung“ und des Verhaltens als Bürger unter Bürgern, als citoyen - und seiner geschichtlichen Dimension, ist eine Verbindung und handelnde Einheit, die nicht umstandslos durch eine ,Identität' im Sinne der Diskurse bestimmt ist, in einer ,Identität' integriert. ${ }^{16}$ Hegel hat sich hier implizit den Ideen von ,Volk', ,Nation' und ,guter alter Ordnung' aus der Romantik seiner Zeit entgegengestellt.

(2) Gegenüber den nach Hegel schon in den Prozessen der "Sittlichkeit" erforderlichen Differenzierungen zeigen sich generelle Diskurse und Forderungen der 'Identität' - und in komplexen Gesellschaften desto mehr - vielmehr in ihrer Rolle als Surrogate von nicht oder noch nicht bzw. nicht mehr adäquaten gelebten Anerkennungen: Surrogate für nicht genügend gelingende, erfahrbare Anerkennungen, die in Entsprechung zu den Verhältnissen und den Anforderungen der Integration wären. ${ }^{17}$ Aus der Weise, wie Hegel die Prozesse der „Sittlichkeit“, die Anerkennungen und die Genese der habituellen citoyen-Gesinnung bestimmt, könnte man kritisch-weiterführende Argumentationen zu rekonstruieren versuchen. ${ }^{18}$ Dies ließe sich zu vier Aspekten der Problemvorsicht gruppieren.

- Mit den Erfordernissen der Neuorientierung nach dem Ende der großen 2-Teilung der Welt, mit den Bedürfnissen, die bisherigen Geschichtswahrheiten und Einordnungen noch einmal neu zur Disposition zu stellen, ergab sich nach den Umbrüchen von 1989/90 in signifikanter Weise eine intensive theoretische Diskussion über ,Erinnerung und ,Vergessen', und sie hat sich meist auch mit den konkreten Fragen der ,Identität verbunden. ${ }^{19}$ Eine erste Problemvorsicht muss insofern dem Denken von ,Erinnerung' und ,Vergessen' in dieser Hinsicht gelten. Die Theoriereflexionen und überhaupt das Denken dabei orientieren sich weithin am ,ich erinnere/vergesse ..., ,du erinnerst/

und habituelle Rechtschaffenheit haben sowie darüber auch ihre politische Partizipation (vgl. Hegel 1821, §§ 207, 253 und 308-311).

16 Die Gemeinschaft des politischen Ganzen hat allenfalls ein verfassungsmäßiges institutionelles Symbol bzw. eine symbolische Verkörperung ihrer Einheits-Souveränität und ihres Wollens, im Staatsoberhaupt (bei Hegel, aus eher pragmatischen Erwägungen: ein erblicher Monarch, konstitutionell eingebunden).

17 Zu einer erforderlichen konkreten Differenzierung in Bezug auf gegenwärtige Aktualisierungen des Konzepts der ,Anerkennung' vgl. die profunden Erörterungen in Kloc-Konkołowicz 2015.

18 Ich verbinde dies mit der Hypothese, dass Hegel unter heutigen Konstellationen den virulenten Diskursen der ,Identität' einen ähnlich ausführlichen Exkurs - ,Anmerkung' - gewidmet hätte wie dem Verhältnis von Staat und Kirche (vgl. Hegel 1821, § 270) oder dem romantischen Konzept von (nichtgesellschaftlicher, antipolitischer) Subjektivität (vgl. Hegel 1821, § 140). In dieser Hinsicht möchte das Nachfolgende gewissermaßen diese bei Hegel selbst nicht vorhandene Anmerkung, deren Gehalt, umreißen.

19 Die Literatur, exponentiell seit den 1990er Jahren, vor allem der Mitte jenes Jahrzehnts angewachsen, ist kaum mehr zu überblicken (für die Breite der Diskussion vgl. nur exemplarisch: Assmann 1992; Haverkamp und Lachmann 1993; Emrich und Smith 1996; Weinrich 1997; Assmann 1999; Ricœur 2000; Welzer 2001; Esposito 2001; Echterhoff und Saar 2002; GoodmanThau 2005). 
vergisst ...' bei Prozessen einer individuellen Lebensvergangenheit. Die im Grunde daran gewonnenen (oder genauer zu sagen: abgelesenen, plausibilisierten) Explikationen werden per Analogie auf kollektive Subjekte und deren Bewusstsein übertragen. Doch ist nicht nur fraglich, wie weit diese Analogisierung überhaupt tragen kann, phänomenal wie strukturell. Vielmehr transportiert dies gemeinhin auch eine sehr starke Implikation, die wohl nur für die individual-lebensvergangenheitliche Repräsentation einer verflossenen Seinswirklichkeit einigermaßen adäquat gelten kann: die Implikation, dass Erinnern und Vergessen sich weithin symmetrisch verhalten, das Erinnerte und das Vergessene materialiter die komplementären Bereiche dessen sind, wie - je zuständlich - das Stattgehabte (bzw. subjektiv als so stattgehabt Erlebte) zu einem gegenwärtigen Bewusstsein und dessen Orientierung gehört. Beim Individuum handelt es sich um die eigene normale Kontinuität eines (Erlebens- und Reflexions-) Bewusstseins wo das wirkliche Vergessen, im Unterschied zum allmählichen Verblassen oder der beständigen Anstrengung der Verdrängung, ohnehin gar nicht so leicht ist. ${ }^{20}$ Es ist hier zunächst einmal die lebensgeschichtlich zusammengekommene Menge, die sich, dabei in jeweiliger Deutung, in das (aktual) Erinnerte und das Vergessene (d.h. das Abgedrängte oder Abgekoppelte des Bewusstseins) teilt. Bei kollektiven Subjekten hingegen und deren Bewusstsein handelt es sich um das ungleich komplexere Verhältnis der GenerationenTradierung (sowie des kollektiven Austauschs). Bei allem, wo jener Tradierungsprozess - sowie für dessen Validierung, eventuelle Erweiterung, Differenzierung oder überhaupt Neuerschließungen: materielle, objektiv-dastehende Überbleibsel einer Vergangenheit die Kette zu einer anderen Quelle denn der Macht der Gegenwart ist, sind das Erinnerte, und was der Bezug darauf bedeutet für die Formung einer ,Identität', nicht die einfache Kehrseite des Vergessenen. In dem, was für kollektive Subjekte sich als ihre geschichtliche ,Identität' darstellt, sind in weit stärkerem Maße, als dies sicher auch für Individuen und ihr Bewusstsein ihrer Lebensvergangenheit zutrifft, alles Fragmentstücke von Konstruktionen, sowohl das ,erinnerte' Geschichts-Bild, d.h. die Gestalt, in der Geschichte präsent ist, als auch das ganze Feld des,Vergessenen', Verlorenen, Verdrängten und dessen Faktoren wie Ökonomie. - Sich diesen ersten Aspekt bewusst zu halten ist vor allem deshalb von Bedeutung für die Diskurse der ,Identität', weil in Zeiten von Umbrüchen und Krisen ein auftretendes neues, alternatives Geschichts-Bild, wenn es gut inszeniert ist und mit der Behauptung neuer,Wahrheiten', die bisher unterdrückt bzw. vorenthalten gewesen seien, relativ leicht Anhängerschaft finden kann. Der Glaube, dass einem etwas verheimlicht werde, ist oft leicht zu wecken. Und das kann ,Identitäts'-Diskurse, wenn sie nicht schon gesellschaftlich stabil sind, schnell in Polarisierungen treiben.

- Substanzialistische (,romantische') Vorstellungen eines ,Volks' (oder ,Nation') und seiner Geschichte - alle Gruppen der Gemeinschaft einheitlich um diese ,Identität' herum 
versammelt - lassen übersehen, dass schon im Individuellen man nicht rein aus sich heraus erfassen kann, was einen im Wesenscharakter ausmache. Das gilt erst recht für kollektive Subjekte, insofern dort die verschiedenerlei Gruppen eines gesellschaftlichen Gefüges und verschiedene sachliche Perspektiven (von privaten Lebensformen bis zur hohen Politik und dem Außenbezug der Gemeinschaft) sicherlich zumindest eine plurale Auffächerung im Verständnis der ,Identität' des Gemeinsamen bedeuten. Substanzialistische Vorstellungen zehren von der Suggestivkraft dessen, als könnte man - im Kollektiven bedeutet das: die ,echten' Volksgenossen, deren Sensorium und Urteilssicherheit nicht von ,Fremdem' korrumpiert oder sediert ist - für sich alleine bestimmen, wer und was man ist und was der eigene Anteil an Interaktionen mit anderen ist, d.h. mit NichtZugehörigen: als könnte man, rein aus dem Inneren heraus, in eigener Hoheit den Charakter seines Wesens als Subjekt gewahren, könnte ,wahrheitlich` empfinden, wenn ein Schwinden oder Gefahren eintreten - und von wem oder was dies verursacht sei -, und überhaupt, dass man allgemein Richter in eigener Sache sein könne. Aber, ,Identität ist niemals etwas Einheitliches und Klares, weder als jeweiliges gegenwärtiges Leben noch als geschichtliches Eines, und ist auch niemals ein Autochthones. Im Gegenteil ist ,Identität', wenn dies etwas Sinnvolles bedeuten soll, wohl stets hauptsächlich ein dynamisches (und durchaus aufgefächertes) Ergebnis, hervorgegangen aus komplexen Prozessen von ,Identitäts'-Kämpfen verschiedener Richtungen, aus ,Identitäts'-Zusprechungen durch äußere (oder übergeordnete) Autoritäten bzw. Mächte, und oft auch aus dem, sich eine ,Identität' umzuhängen (oder zu entlehnen) - sich nobilitieren zu wollen, indem man eine eigene ,Identität' ableitet und legitimiert aus einer bekannten anderen mit hohem Prestige.

- Untersuchungen der gesellschaftlichen Mentalitäten und ihrer inneren Verständigungen haben in der jüngsten Zeit die Fruchtbarkeit des Forschungsprogramms der „Erinnerungsorte“ / "lieux de mémoire“ gezeigt: die „Erinnerungsorte“ / "lieux de mémoire" einer Gesellschaft (einschließlich ihrer subkulturellen Milieus) aufzuarbeiten - deren Pluralität, die ein jeweiliges ganzes Feld bildet. ${ }^{21}$ In den thematischen ,Identitäts'Diskursen jedoch werden die ohnehin problematischen Voraussetzungen ihres Denkens oft durch eine heute ganz neuartige (und durchaus nichtselbstverständliche) gegenteilige Letztentscheidung potenziert. Es ist die Entscheidung, die Konzeption von Erinnerung, Geschichtsbewusstsein und ,Identität' hauptsächlich in Orientierung an dramatischen und moralisch hoch besetzten - Groß-Begebenheiten zu entwickeln. ${ }^{22}$ Dies reproduziert etwas in der Theorie, was zuvor einmal die Weise von, Identitäts'-Konstruktion aus Mythen gewesen war (seien es Mythen in Gestalt von Heroen und Heldentum oder zuweilen auch, genauso funktional, solche von Märtyrertum). - Entstanden ist es als Reflex auf die ganz

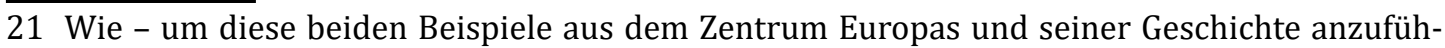
ren - exemplarisch die Diskussionen in Frankreich und Deutschland solche differenzierenden Thematisierungen angegangen sind (vgl. Nora 1984-1992 sowie François und Schulze 2001).

22 In den neueren Theorien (s. oben Anm. 19) vorzugsweise meist aus dem Bereich Holocaust, z.T. auch aus dem Bereich Stalinismus. 
spezifischen verhängnisvollen Erfahrungen - Groß-Erfahrungen - des 20. Jahrhunderts. Doch es bringt, was immer sonst berechtigte Motivationen dieser Exemplifizierung sein mögen, eine fragwürdige Dramatisierung in überhaupt alles Verständnis. Und es verdeckt vor allem tendenziell auch das, was in den alltäglichen Verhältnissen mindestens ebenso prägende Charakteristika des jeweiligen kollektiven Bewusstseins und der vertrauten Lebensgestaltung sind. Für alltägliche ,Identitäten' sowie ,Identitäts'-Verständigungen - sobald einmal das Stadium mythologisch-religiöser Legitimationen abgelegt ist - ist vielmehr jenes Konzept der spezifischen „Erinnerungsorte“ / „lieux de mémoire“, und die eben sind in jeder Gesellschaft und jeder Kultur ein Zusammenwirken von vielen, evidentermaßen weitaus adäquater. Der Alltag darf, das meinte Hegel auch mit seiner Bemerkung zu einem gesunden nüchternen Patriotismus (s.o.), nicht künstlich überlagert, ja aufgeheizt werden mit Heroismus und Märtyrertum (bzw. ,Identitäts'-Erinnerungen daran). Und solche „Erinnerungsorte“ / „lieux de mémoire“, nämlich insofern sie konkret sind, lassen sich auch kritisch reflektieren. ${ }^{23}$

- Schließlich noch ein letzter, aber entscheidender Punkt. In der gesellschaftlichen und intellektuellen Rede über,Identität 'wird, und gerade wo es um Probleme der Gegenwart geht, auch meist so generell gesprochen, dass leicht übersehen wird, dass genau Probleme nicht durchweg überall sich in gleichem Grad und Dringlichkeit darstellen. In oberen und weiter umfassenden Bereichen zum Beispiel - bei den Menschenrechts-Prinzipien der UN usw., oder in den Ländern der Europäischen Union etwa bei den Instanzen und der Politik dieser Gemeinschaft - hat sich in den Menschen im Allgemeinen etwas ergeben, das eine Art intellektuelles Bekenntnis dazu ist; dass darüber Diskurse geführt werden und oft Kompromisse geschlossen werden müssen, ist dort nichts Irritierendes, ja ist durch die Medien öffentlich präsent. Und in den unmittelbaren umgebenden Bereichen, im Regionalen (bes. von Herkunft und Lebenstätigkeit) ist man normalerweise so verwurzelt, dass man Ambivalenzen zulassen kann (d.h. sofern nicht abrupte Veränderungen eintreten). Das Problematische liegt dagegen bes. auf gleichsam mittlerer Ebene - bes. schwierig sind ,Identitäten' und ,Identitäts'-Erfahrungen auf mittlerer Höhe bzw. Reichweite, wie etwa bei Nationen und historischen Großlandschaften. Diese Einheiten brauchen, und zumal in Krisen, einen hohen Identifizierungs-Bedarf; und dort wird dann in der Weise der Rede und Weise der politischen Aktion leicht übersehen - oder sogar absichtsvoll unterdrückt -, dass es in Bezug auf höhere Zugehörigkeiten (und der Prinzipien) wie im Lokaleren bereits flexible Kompetenzen gibt.

Diese vierfachen Reflexionen berücksichtigt, könnte das Thema der ,Identität,' als differenziert zu verortendes Begleitphänomen gesellschaftlicher VerständigungsProzesse in Phasen großer Affirmationsunsicherheit und Orientierungsbedürfnisse, durchaus auch eine Bedeutung in einer Theorie moderner politischer Ordnungen

23 Mythen des Autochthonen hingegen stilisieren meist stark die Gründungs-,Identitäten, in Narrationen einer Ursprungs- oder Gründungs-Geschichte. (Da werden dann auch häufig Tatsachen erfunden, Urkunden bzw. Belege gefälscht.) 
wie ihrer politischen Bewegungen einnehmen: theorieseitig, nicht nur sozusagen sozialpsychologisch. Das Thema wäre dann zurückgebaut auf einen Kern, worin das Narrative (narrative Konstruktionen) und die durch Praktiken, bes. Einsozialisierung vermittelten Formen ohne blinde heroisierende Affirmation und Überdeckung innergesellschaftlicher (bzw. gruppierungsimmanenter) Probleme wie Aufgaben gedacht werden können. ${ }^{24}$ Eingebunden in Hegels Konzept der "Sittlichkeit“ und ihrer Differenzierungen, könnte das Thema der ,Identität', seine erscheinende Gestalt und sein Grad, zum Index eines in den Verhältnissen und Institutionen - zeitbedingt verstehbar - unbefriedigten gesellschaftlichen Bewusstseins werden. Ohne Reflexion aber wird es infektiös, und Spielball von Identitätspropaganda und Geschichtspolitik.

\section{Der Halt im Netz der Geschichte (Der Problemdruck der großen Umbrüche)}

Geschichte schafft Legitimitäten, schafft Identifikationen, und schafft Ziele. Allgemein: Der Bezug auf Geschichte vermittelt einen Stand der sozialen und kulturellen Integration. Das gilt für ,normale', geregelte Zeiten wie für Zeiten großer Veränderungen und eines davon ausgehenden Problemdrucks. Darum sind entstehende Reflexionen über Erinnerungen, über die Geschichtskultur einer Gemeinschaft, stets Ausdruck eines drohenden Abbrechens der Überlieferungen; oder Ausdruck des Bewusstseins, mit der geschichtlichen Dynamik evtl. nicht mehr Schritt zu halten, so dass die produzierten Wissensbestände und Deutungen tot bleiben oder ins Leere laufen. In Zeiten besonderer Umbrüche werden dabei die Erbschaften der jüngsten Vorgeschichte neu zur Betrachtung und Beurteilung freigegeben.

Die Ereignisse von und seit 1989/90 waren im eminenten Maße ein Auslöser dieser auch mentalen Unruhe und Orientierungsvergewisserungen. In Gesellschaften mit sicherem, langherkommendem gesellschaftlichem Bewusstsein, Bewusstsein ihrer pluralistischen Wirklichkeit - sicher und z.T. auch durch frühere Erfahrungen mit sich selbst aufgeklärt über mögliche Untiefen und Verführungen im Eigenen - kann man die Prozesse begleitet sehen von den genannten theoretischen Verständigungen über das Wesen von ,Erinnerung' und ,Vergessen' und ihre Faktoren der Konstruktion; begleitet zudem meist von einer bezeichnenden Erneuerung der Gesellschaftstheorie, eine parallele Debatte über Gesellschaftlichkeit und ihre moderneentsprechenden, nicht nur traditionalistischen Formen. In Gesellschaften aber, in denen das Empfinden herrscht, sich überhaupt von bisherigen Irrwegen, bes. oktroyierten oder verordneten Falschbildern befreien zu müssen und die eigene Geschichte überhaupt erst wieder zu erschließen,

24 In dieser Situierung könnte mit Hegel wohl auch ein - relational - positiver Diskurs der ,Identität' begründet sein: ,Identität' (und Identitätspolitik) als Identitätsfindung - und stärkendes Gruppen-Selbstbewusstsein - von bisher Marginalisiertem oder Verleugnetem innerhalb einer Gesellschaft (nationale Minderheiten; von einer Zentralmacht erstickte Regionalitäten; nicht-standardmäßige sexuelle Orientierungen; usw.). 
in Souveränität über die eigene Geschichte, werden die Diskurse der ,Identität' meist ungleich schillernder.

Das gilt offenkundig für viele der Gesellschaften der einst sowjetisch beherrschten ganzen Hemisphäre der Welt, die ein vormals durch Ideen von transnationaler ,sozialistischer Verbrüderungs- und Solidaritätsgemeinschaft dominiertes gesellschaftliches, politisches und geistiges System zu verarbeiten hatten. Je neuer ein postsozialistisches System, je weniger schon gefestigt die demokratische Kultur, je weniger schon pluralitäts- und konflikterfahren, desto entfesselter ist in solchen Ländern oft der Diskurs über ,Identität', ja konstruierte Mythen der Verständigung, und desto mehr ist der Bezug auf Geschichte national aufgeladen. In stark sich wandelnden Gesellschaftsordnungen, und wenn noch keine festen Bestände eines Konsenses über die eigene ,Identität' und ihre Tradition, kommt es zum Kampf um die ,Wahrheit', Kampf um die nationale Deutungshoheit, auch zu Visionen und Versprechungen an das kollektive Wahlvolk-,Wir'. Im Erfordernis, nach dem Ende der Über-Geschichte von der Einheit und Gemeinschaft der ,sozialistischen Bruderstaaten' erstmals das eigene gesellschaftliche sowie nationale Sein zu thematisieren - eine eigene ,Identität' und Geschichtsverortung gegen die bisherigen Fremdnarrationen -, verselbständigen sich allzu leicht substanzialistische Projektionen eines nationalen oder ethnischen ,Wesens: Und an den verschiedenen Lagern der ,Identitäts'-Angebote wiederum spaltet sich die Gesellschaft, entsolidarisiert sich in sich selbst.

Die Vorstellung von einer autonomen, gar einfachen ,Identität' ist jedoch allemal eine prekäre Ware im mentalen Haushalt von Gesellschaften und ihren Verständigungen. ,Identitäten' sind immer mit bestimmtem Geschichtsbewusstsein verbunden, und was dies Geschichtsbewusstsein von Gesellschaften und Subgruppierungen ausmacht, steht, wo eine erfahrungsbasierte wirkliche Reife und Resilenz gewonnen ist, normalerweise in einem zwiefachen Bezug auf Geschichte. Identitäten, als jeweilige ,Identitäts'-Konstruktionen, verankern sich nicht nur - und stabilisieren sich - an Geschichtlichem, an historischen Identitäts-,Wahrheiten! Sondern in Reife stehen sie auch in einem gewissen dynamischen Bezug, sich auch auf die zugrundeliegenden geschichtlichen Wissensurteile hin zu überprüfen und sich durch bisher noch nicht bedachte Perspektiven oder noch nicht Verstandenes in Frage stellen zu lassen. In der Moderne seit dem 20. Jahrhundert ist dies oft die aus Selbstbewusstsein erwachsene kritische Arbeit an eigenen Mythen. - Undramatisch gehört das dann normalerweise mit dazu, als Ergänzungen und Erweiterungen, als Differenzierungen und gewisse Korrekturen. In problematische Entwicklungen kommt dies vielmehr, wenn gegen Verunsicherungen, die in Zeiten großer Umbrüche entstehen, neue feste Eindeutigkeiten gezeichnet werden - wenn ,Identitäts'Bedürfnisse zum Vorherrschenden werden und wenn eine (offene oder unterschwellige) Erinnerungs-Politik betrieben wird.

Tendenzen, dass dabei die negative Seite zum treibenden Faktor wird und imaginierte - und instrumentalisierbare - Furcht und Bedrohung, ohne dies mit der 
generellen globalen Modernisierung in Verbindung zu bringen, zum Bestimmenden werden, d.h. mentale Personalisierungen (resp. Re-Personalisierungen) von Verursachern, lassen sich in vielen Ländern beobachten. Aber das macht es nicht besser. Das Faktum des Ubiquitären ist keine Rechtfertigung. - Ein Blick nach nebenan, auf spezifisch die unmittelbaren Nachfolgestaaten der ehemaligen Sowjetunion mag hier abschließend Gefahren wie Perspektiven dieser heute virulenten Dynamik deutlich machen. Diese Länder haben in besonderer Weise eine potenzierende Konstellation. Genauer ist es eine doppelte zusätzliche Problematik. Zum einen ist bei ihnen auch ihr jeweiliges Territorium, und damit der Umfang des unter einer Identität von Gemeinschaftlichkeit zu Befassenden, das eines unter einer vormaligen, nun nicht mehr bestehenden Ratio Geschaffenen: das der in der Sowjetunion gebildeten Republiken. Die darin lebenden Menschen sind weder ethnisch noch in den meisten Fällen allgemein kulturell (sowie religiös) homogen; aber alle sind zunächst einmal gleichberechtigte Bürger dieser neuen Staaten, bzw. müssen dies bleiben. Sie dürfen nicht durch die jeweilige neue ,Identität' gegeneinander gewertet oder gar polarisiert werden. Zum anderen scheint die Geschichte dort auf den ersten Blick tatsächlich nur eine einzige Richtung, einzige Option als das national und moralisch Notwendige zu weisen. Sicher geht es um ein Rückgängigmachen vormaliger Strukturen, rückgängig gegen die Sowjetifizierung, welche in vielen Phasen der 70 Jahre der sowjetisch dominierten Hemisphäre der Welt dort im besonderen Maße auch die ,Russifizierung' bedeutete. Doch wenn nun eine eigene, neue Identität zur Aufgabe wird, darf nicht der negative Affekt, die Anti-Haltung gegen die vorherige Geschichte wiederum jede offene zivilgesellschaftliche Verständigung über das Eigene, die Gegenwart und ihre pluralen Lebensverwurzelungen niederdrücken. Die den Zusammenhalt, die Loyalität und die Orientierung stiftende Identität der Gemeinschaft darf nichts neues Einfaches suchen. Das wäre nur die Wiederholung der Geschichte, einfach unter umgekehrten Vorzeichen.

Beides sind, oft unbewusst, problematische Schatten der Vergangenheit. Doch sind diese Staaten zugleich in einer eigentlich günstigen Lage. Sie können von anderen Ländern lernen, die z.T. bereits früher durch Erfahrungen mit Chancen und Gefahren einer postsozialistischen gesellschaftlichen Orientierung und Identität hindurchgegangen sind (in Europa in den Regionen des ehemaligen Jugoslawiens dramatisch bis hin zum Krieg). Sie können aber vor allem allgemein von den ,westlichen', schon länger demokratisch und pluralistisch verfassten Gesellschaften, für die die Umbrüche von 1989/90 ebenfalls ein Einschnitt in ihren bisherigen Verständigungen und Orientierungen waren, lernen: dass nicht in einem abgehobenen ,kulturalistischen' Raum ein Diskurs über ,Identität‘ und Geschichtsbewusstsein aufgemacht werden darf, der sich sonst leicht verabsolutiert. Sondern dies muss eingebunden sein in parallele gesellschaftstheoretische und sozialpsychologische Betrachtungen über die betreffenden Bedürfnisse der Versicherung und die konkreten realen Ursachen. Und schließlich kann auch von den in vielen Ländern bereits erreichten Einrichtungen gelernt werden, prägende Geschichtsereignisse nicht nur der historischen Deutung und Bedeutungsgebung im jeweiligen eigenen nationalen 
Horizont zu überlassen, sondern in bilateralen, gemeinsamen Kommissionen die wechselseitigen Erinnerungen zusammenzubringen und zu versöhnen - den Blick der Anderen ebenso zu Wort kommen zu lassen, bis in die Schulbücher hinein. Wo man sich diesen Möglichkeiten verschließt, droht allenfalls eine neue 2-Teilung der Welt, nun eine Teilung in der Mentalität von Geschichtsbewusstsein und ,Identitäts'-Verständnis; auch eine 2-Teilung innerhalb dieser Gesellschaften zwischen Jüngeren und Alten.

Das Bild, das Hegel von der „Sittlichkeit" eines gesellschaftlichen und staatlichen Zusammenhalts verdeutlichte - auch in Erwartung eines Sichausbreitens über die Welt -, war hier gewissermaßen schon vielem aus unserer heutigen Zeit voraus: Anerkennung des Verschiedenen lernen; nicht überall innere Feinde und ,Unnationales‘ wähnen; nicht vom Krieg und von der Selbstbehauptung unter Bedrohung her denken; und nicht auf verbissene ,Identitäts'-Kämpfe auszugehen, die fast stets die einer bestimmten Gruppe, die mit ihrer Auslegung, ihrer Definition zu Einfluss zu gelangen sucht, sind - und oft auch nur Stellvertreter- oder Ablenkungskämpfe. Doch das scheint manchmal nicht so einfach. In dieser Hinsicht sind wir wahrscheinlich realistischer als Hegel. Realistischer, als Hegel dies bereits sein konnte. ,Hegelianer*in' zu sein muss auch heißen, nicht bei Hegel stehenzubleiben.

\section{Literatur}

Adolphi R. 2018. „Übersoziale Kriterien in der Theorie sozialer Geltungen. Zur pluralistischen Logik der hegelschen ,System'-Form des objektiven Geistes", in: T. Oehl und A. Kok (Hg.), Objektiver und absoluter Geist nach Hegel (S. 157-190). Leiden - Boston: Brill.

Adolphi R. 2019. „Pokusy rozumu. Romantyzm w tradycjach europejskiej

Nowoczesności - na przykładzie Niemiec i Polski“ [Die Versuchungen der Vernunft. Romantizismus in den Traditionen der europäischen Moderne - das Beispiel Deutschland und Polen], in: E. Nowak (Hg.), Recepcja i oddziaływanie Marka Siemka w Europie (S. 365-391). Warszawa: Wydawnictwa Uniwersytetu Warszawskiego.

Assmann A. 1999. Erinnerungsräume. Formen und Wandlungen des kulturellen

Gedächtnisses. München: C.H. Beck.

Assmann J. 1992. Das kulturelle Gedächtnis. München: Beck.

Echterhoff G. und Saar M. 2002 (Hg.). Kontexte und Kulturen des Erinnerns. Maurice Halbwachs und das Paradigma des kollektiven Gedächtnisses. Konstanz: UVK Verlagsgesellschaft mbH.

Emrich H. M. und Smith G. 1996 (Hg.). Vom Nutzen des Vergessens. Berlin: AkademieVerlag. 
Esposito E. 2001. La memoria sociale. Mezzi per comunicare e modi di dimenticare. Roma - Bari: GLF editori Laterza.

François É. und Schulze H. 2001 (Hg.). Deutsche Erinnerungsorte. 3 Bände. München: Beck.

Goodman-Thau E. 2005 (Hg.). Das eigene Erinnern. Wien: Passagen-Verlag.

Haverkamp A. und Lachmann R. 1993 (Hg.). Memoria. Vergessen und Erinnern. München: Fink.

Hegel G. W. F. 1807. Phänomenologie des Geistes. Bamberg - Würzburg: Goebhardt. Hegel G. W. F. 1819/20 [1983]. Philosophie des Rechts. Die Vorlesung von 1819/20 in einer Nachschrift. Hg. von D. Henrich. Frankfurt am Main: Suhrkamp.

Hegel G. W. F. 1821 [erschienen 1820]. Grundlinien der Philosophie des Rechts. Naturrecht und Staatswissenschaft im Grundrisse. Berlin: Nicolaische Buchhandlung. Zitiert nach der Ausgabe: Hegel G. W. F. 1970. Werke. In 20 Bänden. Hg. von E. Modenhauer und K. M. Michel. Bd. 7 [dort auch mit Mitschriften aus Hegels Vorlesungen über dieses Lehrbuch]. Frankfurt am Main: Suhrkamp.

Hegel G. W. F. 1830. Encyclopädie der philosophischen Wissenschaften im Grundrisse. Dritte Auflage. Heidelberg: Oßwald. - Zitiert nach der Ausgabe: Hegel G. W. F. 1970. Werke in 20 Bänden. Hg. von E. Modenhauer und K. M. Michel. Bd. 10 [Dritter Teil. Philosophie des Geistes]. Frankfurt am Main: Suhrkamp.

Kloc-Konkołowicz J. 2015. Anerkennung als Verpflichtung. Klassische Konzepte der Anerkennung und ihre Bedeutung für die aktuelle Debatte. Würzburg: Königshausen \& Neumann.

Locke J. 1694. An Essay Concerning Humane Understanding. $2^{\text {nd }}$ Edition. London: Awnsham and John Churchil[1].

Nora P. 1984-1992 (Hg.). Les lieux de mémoire. 3 Bände. Paris: Gallimard.

Ricœur P. 1983-1985. Temps et récit. 3 Bände. Paris: Éd. du Seuil.

Ricœur P. 1990. Soi-même comme un autre. Paris: Éd. du Seuil.

Ricœur P. 2000. La mémoire, l'histoire, l'oubli. Paris: Éd. du Seuil.

Sen A. 2006. Identity and Violence. The Illusion of Destiny. New York: Norton. [Dt. 2007.

Die Identitätsfalle. Warum es keinen Krieg der Kulturen gibt. München: Beck.]

Weinrich H. 1997. Lethe. Kunst und Kritik des Vergessens. München: Beck.

Welzer H. 2001 (Hg.). Das soziale Gedächtnis. Hamburg: Hamburger Ed. 